\title{
HOW TO FUSE OPTICAL AND RADAR IMAGERY?
}

\author{
Gintautas Palubinskas \\ German Aerospace Center DLR \\ Remote Sensing Technology Institute \\ Oberpfaffenhofen, 82234 Wessling, Germany \\ Gintautas.Palubinskas@dlr.de
}

\begin{abstract}
Information extraction from multi-sensor remote sensing imagery is an important and challenging task for many applications such as urban area mapping and change detection. There exist a lot of publications on this topic, but quite often they follow the same strategy: put all data into one stack and search for the best classifier for one particular example. Transfer of the results to other examples quite often fails. Especially during optical and radar data fusion different aspects and factors such as different acquisition geometries, incommensurable nature of features and many other parameters should be accounted for. In this paper we shall present a general workflow for optical and radar/SAR data fusion and discuss the main problems occurring when applying it for classification applications.
\end{abstract}

Index Terms - Multi-sensor fusion, orthorectification, co-registration, classification, change detection

\section{INTRODUCTION}

Data fusion is a rapidly developing topic in various application areas during the last decades. Image fusion in remote sensing is one of them. However fusion of different sensor data such as optical and radar imagery is still a challenge. In this paper the term 'radar' is equivalent to Synthetic Aperture Radar (SAR). Different modalities of data can be obtained by different sensors for the same area, and more properties can be revealed on the area structure, contents and properties. Incommensurability of different sources of data (e.g. optical, SAR, and DEM) requires a proper design of fusion process. For example in $[1,2]$ statistical versus neural network approaches for multisensory data fusion and classification are investigated. Linear and logarithmic opinion pools optimized by multilayer neural network are proposed for combination of multisensory data (multispectral, elevation, slope, aspect, and SAR). Several approaches for multisensory data fusion following consensus theory and employing different techniques such as Bayesian networks, neural networks and fuzzy logic approaches were developed e.g. see results of fusion contest [5] or urban area classification [3, 4].

We approach the joint optical and radar data classification task using a more general multi-sensor view on the whole data fusion problem [10]. Thus data acquisition planning and pre-processing become very important steps for a successful data fusion. Additionally, we discuss some problems of classification arising from different acquisition geometries of data.

The paper is organized as follows. First, in Section 2 we present a general processing chain for multi-sensor data fusion. Then, in Sections 3 and 4 some of the aspects and parameters significantly influencing the classification accuracy and building signature in SAR orthoimage are discussed. The paper ends with conclusions, acknowledgments and references.

\section{PROCESSING CHAIN}

A proper preparation of data is a very important prerequisite for a successful data fusion. Following sub-sections present our approach methodologically including the data processing chain [10]. The optical and SAR data processing chain is presented in Figure 1.

Below is the detailed explanation of separate steps:

- Input image data are of multi-sensor nature. Two acquisition geometries are possible:

- Accidental acquisition geometry

- Orthogonal acquisition geometry [8]

- Pan-sharpening of optical multispectral data using panchromatic band to enhance pixel resolution simultaneously preserving spectral characteristics [9]

- Despeckling of SAR data is optional [11]

- Additional data (marked in red) are used:

- Digital Elevation Model (DEM) for georeferencing

- Reference data for ground control points (GCPs) und training/test data extraction

- Orthorectification of imagery using available DEM (e.g. SRTM) 
- Manual or automatic co-registration of multisensor images [13]

- Feature extraction from input images e.g.

$$
\text { ○ Gabor texture }
$$

- Fusion und classification methods e.g.:

$$
\begin{array}{ll}
\circ & \text { Maximum likelihood (ML) } \\
\circ & \text { Neural networks (NN) } \\
\circ & \text { Support vector machines (SVM) } \\
\circ & \text { INFOFUSE [7] }
\end{array}
$$

- Classification accuracy assessment by calculating confusion matrices for test data

Here we have to note that a simple stacking of all available data (e.g. optical and radar) and their features and then applying one of the above mentioned classifiers for the fusion can result in unstable results especially for urban area mapping. For example, sometimes the addition of radar information to optical data increases the classification accuracy, but quite often the quality decrease is observed [4]. We try to explain the reasons for such instability or randomness of urban area classification in the following sections. Additionally, we will discuss some of the aspects and parameters significantly influencing the classification accuracy.

\section{ASPECTS/PARAMETERS INFLUENCING CLASSIFICATION ACCURACY}

Below is the list of aspects/parameters which can influence the quality of data fusion or classification accuracy significantly.

\subsection{Data acquisition/selection}

Different acquisition time of data

- Year (seasonal changes and other changes are possible)

- Month (seasonal changes are possible)

- Weather conditions during acquisition can have influence on data

Test site

- Type

Sensor

Location

- Type

- Bands

- Acquisition geometry $[8]$

\subsection{Georeferencing/Orthorectification}

Orthorectification result is highly influenced whether a Digital Terrain Model (DTM) or Digital Surface Model (DSM) is used especially for side looking sensors (see section 4). Availability of DSM is of great importance for a good data quality. Additionally, DSM allows simulation based analysis, e.g. extraction masks for sensor shadow, layover, etc. [14], thus allowing to remove ambiguous regions in a resultant orthoimage. Moreover, usage of SAR (TerraSAR-X) high location accuracy can be used to enhance optical sensor models [12].

\subsection{Multi-sensor data co-registration}

Though orthorectification is performed perfectly there remain some residual errors mainly shifts when overlaying different images. Manual co-registration method suffers from the subjectivity of GCPs selection. There exist some automatic methods, e.g. based on mutual information [13], but the question is which one to select? Further question is how to set the parameters?

\subsection{Feature extraction}

Feature extraction is performed in order to describe a scene (world) under investigation quasi completely. Here a set of questions should be answered. Is despeckling of SAR data is necessary? Which features should be extracted? Texture? Should feature selection performed? Should original data geometry be used e.g. slant range for SAR data? Should radiometric normalization be performed?

\subsection{Clustering}

Feature extraction step can produce a huge amount of data with different nature. Clustering of features is useful for bringing incommensurable data to common domain and simultaneously for data reduction. The number of clusters should be defined automatically depending on scene complexity [6].

\subsection{Classification and fusion}

Here the real data fusion is happening. Again a lot of questions should be answered. Which method should be used? Which parameters? How training/test data should be selected? How to manage different sizes of classes? Is definition of sub-classes necessary? Does usage of different features for different classes increase the accuracy?

\subsection{Quality assessment}

Different methods produce different results. The evidence of data fusion should be demonstrated clearly. Here is a set of questions. How test data for validation should be selected? Which measures: overall, per-class etc. should be used? How validation should be performed?

\subsection{Software}


Nowadays such a processing chain is implemented in some software in order to run on computer. Which software is good and can be used or should we write our own?

\section{BUILDING SIGNATURE IN SAR ORTHOIMAGE}

In order to understand better e.g. how a building is projected in a radar orthoimage one must look in detail at SAR image formation process as shown in Figure 2. Green box shows the projection of SAR image using DEM whereas orange box - DSM. It is assumed that a building with a gable roof (yellow color) is standing on a flat ground (green line).

Green box shows how parts of a building are projected on the ground. Starting analysis from left to right, first come two layover areas (L1, L2, red color), each composed of three different contributions from building and ground (mixed radar signature). Then follow the right roof ( $R$, blue), which is the only true radar signature but projected in a wrong location. Finally, comes a shadow area (S1, S2), where eventually other neighboring buildings can be projected. So for this type of building only a small part of the roof (right roof) is unambiguously projected on the ground but unfortunately in the wrong place. So the naive fusion of such SAR orthoimage with optical data makes no sense or will deliver random and unstable results for such type buildings. Other objects will produce other patterns thus individual analysis is needed.

Orange box shows how parts of a building are projected on the ground using DSM or 3D model for orthorectification. First two layover areas are identical as in the previous case. Then come two interpolated copies of a first layover (L1) and one interpolated copy of a second layover (L2). Layover area is almost two times larger than in the previous case. The roof part is smaller (interpolated) in this case and is only partly projected in the correct location. Shadow areas (S2) are the same. Again, such orthoimage is not much more useful for the classification purposes than in the previous case, only a small part of a roof is displayed in approximately correct location. Illustration is performed for simplicity in $1 \mathrm{D}$ case. $2 \mathrm{D}$ case will be much more complex to interpret or understand.

\section{CONCLUSIONS}

We hope that our experience and this discussion on some of the aspects and parameters of optical and radar remote sensing data fusion for classification applications will help the readers and scientists in this research area to produce more sound and technically correct results. Moreover, some open issues will stimulate further scientific research.

\section{ACKNOWLEDGMENT}

We would like to thank DigitalGlobe and European Space Imaging (EUSI) for the collection and provision of
WorldView-2 scenes over Munich city. TerraSAR-X data were provided by DLR through the Science Project MTH0948.

\section{REFERENCES}

[1] J.A. Benediktsson, P. Swain and O. Ersoy, "Neural network approaches versus statistical methods in classification of multisource remote sensing data," IEEE Transactions on Geoscience and Remote Sensing, 28(4), pp. 540-552, 1990

[2] J.A. Benediktsson, J. Sveinsson and P. Swain, "Hybrid consensus theoretic classification," IEEE Transactions on Geoscience and Remote Sensing, 35(4), pp. 833-843, 1997.

[3] M. Fauvel, J. Chanussot and J.A. Benediktsson, "Decision fusion for the classification of urban remote sensing images," IEEE Transactions on Geoscience and Remote Sensing, 44(10), pp. 2828-2838, 2006.

[4] A. Makarau, G. Palubinskas and P. Reinartz, "Discrete graphical models for alphabet-based multisensory data fusion and classification," In: Proc. of International Symposium on Image and Data Fusion, 9-11 August, 2011, Tengchong, China, 2011.

[5] F. Pacifici, F. Del Frate, W. Emery, P. Gamba and J. Chanussot, "Urban mapping using coarse SAR and optical data: Outcome of the 2007 GRSS data fusion contest," IEEE Geoscience and Remote Sensing Letters, 5(3), pp. 331-335, 2008.

[6] G. Palubinskas, "An unsupervised clustering method by entropy minimization," In: Maximum Entropy and Bayesian Methods, W. von der Linden et al., Eds., Kluwer Academic Publishers, Netherlands, pp. 327-334, 1999.

[7] G. Palubinskas and M. Datcu, "Information fusion approach for the data classification: an example for ERS-1/2 InSAR data," International Journal of Remote Sensing, 29(16), pp. 4689-4703, 2008 .

[8] G. Palubinskas, P. Reinartz and R. Bamler, "Image acquisition geometry analysis for the fusion of optical and radar remote sensing data," International Journal of Image and Data Fusion, 1(3), pp. 271-282, 2010.

[9] G. Palubinskas and P. Reinartz, "Multi-resolution, multi-sensor image fusion: general fusion framework," In: Proc. of Joint Urban Remote Sensing Event JURSE, 11-13 April, 2011, Munich, Germany, pp. 1-4.

[10] G. Palubinskas, A. Makarau and P. Reinartz, "Information extraction using optical and radar remote sensing data fusion," In: Proc. ASPRS Annual Conference, 19-23 March, 2012, Sacramento, California, USA, 2012.

[11] G. Palubinskas, "Spectral speckle filter for SAR imagery," In: Proc. IGARSS, 22-27 July, 2012, Munich, Germany, 2012.

[12] P. Reinartz, R. Müller, P. Schwind, S. Suri and R. Bamler, "Orthorectification of VHR optical satellite data exploiting the geometric accuracy of TerraSAR-X data," ISPRS Journal of Photogrammetry and Remote Sensing, 66, pp. 124-132, 2011.

[13] S. Suri and P. Reinartz, "Mutual information based registration of TerraSAR-X and IKONOS imagery in urban area," IEEE Transactions on Geoscience and Remote Sensing, 48(2), 939-949, 2010.

[14] J. Tao, G. Palubinskas, S. Auer and P. Reinartz, "Interpretation of SAR images in urban areas using simulated optical and radar images," In: Proc. of Joint Urban Remote Sensing Event JURSE, 11-13 April, 2011, Munich, Germany, pp. 41-44, 2011. 


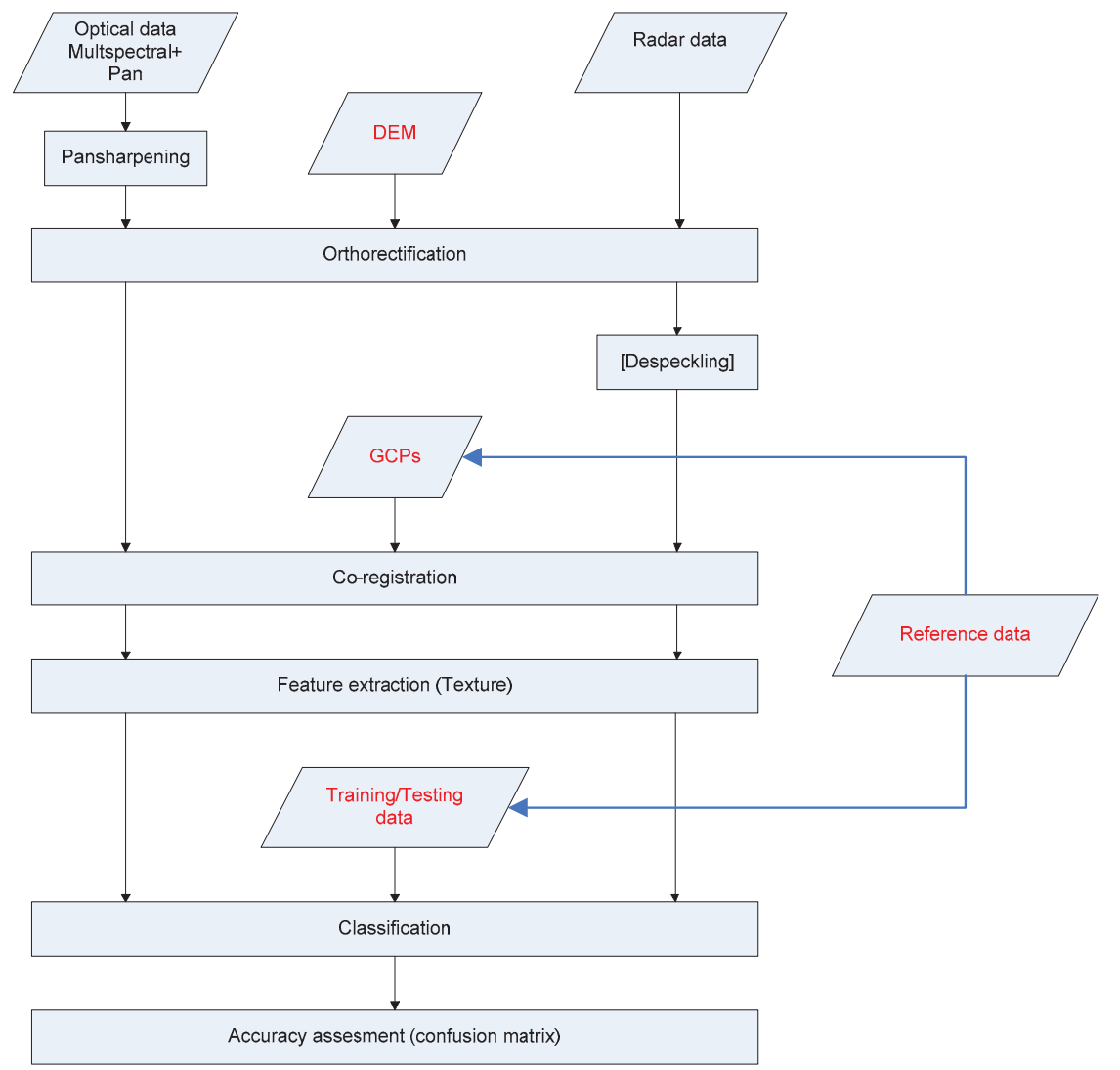

Figure 1. Optical and SAR data processing chain.

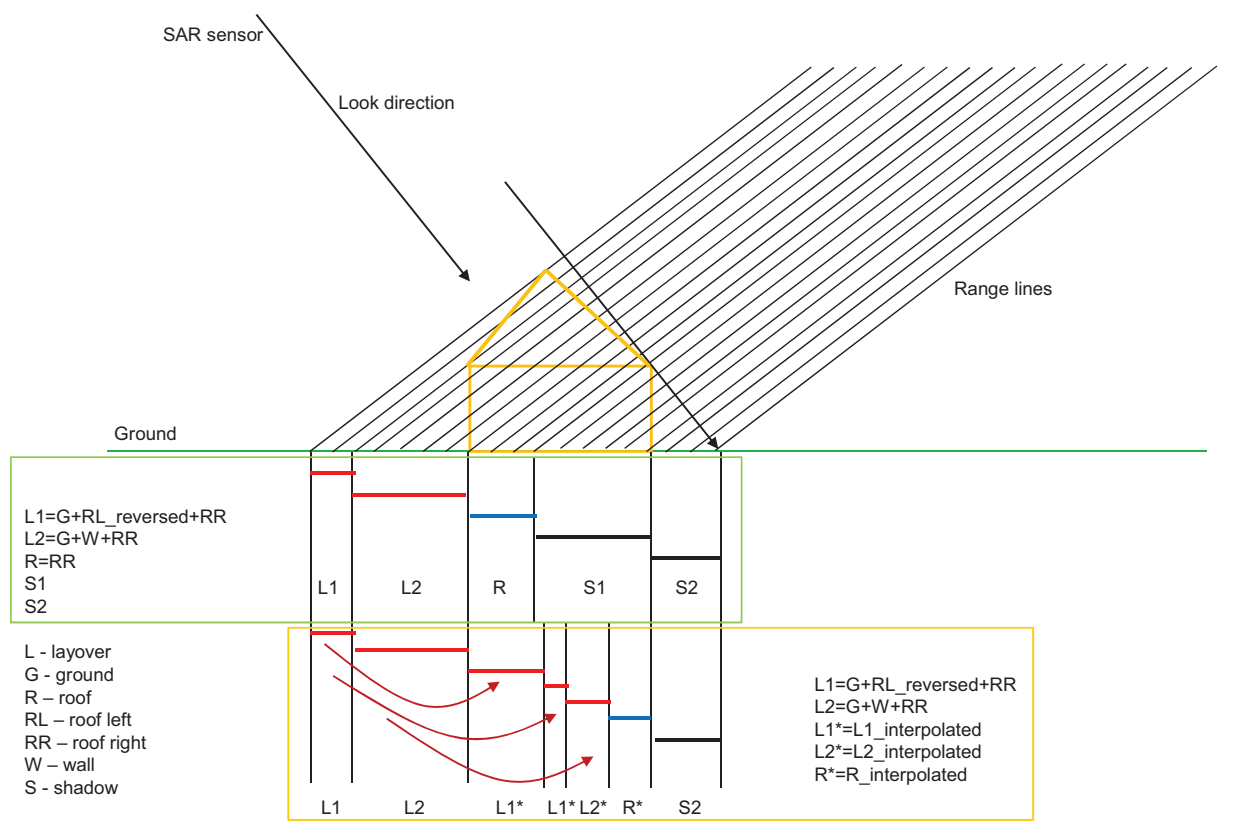

Figure 2. Example of building projection in SAR orthoimage using DEM (green box) and DSM (orange box). 